\title{
Tamanho de amostra e relações lineares de caracteres morfológicos e produtivos de crambe
}

\author{
Sample size and linear relations of the morphological characters and production of crambe
}

\author{
Alberto Cargnelutti Filho ${ }^{\mathrm{I}^{*}}$ Marcos Toebe $^{\mathrm{II}}$ Tatiani Reis da Silveira $^{\mathrm{II}}$ Gabriele Casarotto $^{\mathrm{III}}$ \\ Fernando Machado Haesbaert ${ }^{\mathrm{III}}$ Sidinei José Lopes ${ }^{\mathrm{I}}$
}

\section{RESUMO}

\begin{abstract}
Os objetivos deste trabalho foram determinar o tamanho de amostra para a estimação da média de caracteres morfológicos e produtivos de crambe (Crambe abyssinica Hochst) e avaliar as relações entre esses caracteres. Foi conduzido um experimento a campo em Santa Maria, Estado do Rio Grande do Sul (latitude $29^{\circ} 42^{\prime}$ 'S, longitude 53⒋' W e $95 \mathrm{~m}$ de altitude), no ano de 2008. Na colheita, foram selecionadas, aleatoriamente, 75 plantas e foram mensurados os caracteres morfológicos (altura de planta, número de rácemos por planta e comprimento médio dos rácemos por planta) e os produtivos (número de frutos por planta, número de frutos por rácemo e produtividade de grãos). Foi calculado o tamanho de amostra de cada caráter e investigada a relação entre os caracteres por meio de diagramas de dispersão, análises de correlação, de trilha e regressão. Na cultura de crambe, para estimar a média com uma mesma precisão, o tamanho de amostra (número de plantas) dos caracteres produtivos é maior que o dos morfológicos. O número de frutos por planta tem relação linear positiva com a produtividade de grãos $e$ pode ser utilizado para seleção indireta.
\end{abstract}

Palavras-chave: Crambe abyssinica Hochst, planejamento experimental, dimensionamento de amostra, modelagem, seleção indireta.

\section{ABSTRACT}

The aim of this research was to determine the sample size to estimate the average of the morphological and productive traits of crambe (Crambe abyssinica Hochst) and assess the relationships among these traits. An experiment was conducted in Santa Maria, Rio Grande do Sul State, Brazil (latitude $29^{\circ} 42^{\prime} \mathrm{S}$, longitude $53^{\circ} 49^{\prime} \mathrm{W}$ and $95 \mathrm{~m}$ altitude) in the year 2008. At harvest, 75 plants were randomly selected and measured for morphological traits (plant height, number of racemes per plant and length average of racemes per plant) and productivity (number of fruits per plant, number of fruits per raceme and grain yield). It was calculated the sample size of each trait and studied the relationship among the traits by scatter plots, correlation, path analysis and regression. In the culture of crambe, the sample size (number of plants) of productive traits is greater than morphology to estimate average and it has the same precision. The number of fruits per plant has a positive linear relationship with grain yield and can be used for indirect selection.

Key words: Crambe abyssinica Hochst, experimental planning, sample dimensioning, modeling, indirect selection.

\section{INTRODUÇÃO}

Em experimentos agrícolas, a mensuração em todas as plantas (indivíduos) da área útil da unidade experimental é a técnica adequada para estimar a média do caráter em avaliação. Entretanto, a disponibilidade de tempo, mão-de-obra, recursos financeiros e humanos, muitas vezes, limita a avaliação de grande número de plantas, principalmente, quando muitos caracteres devem ser mensurados. Nesses casos, a amostragem de plantas dentro da unidade experimental é uma alternativa adequada.

O tamanho de amostra é diretamente proporcional à variabilidade dos dados e ao grau de

'Departamento de Fitotecnia, Centro de Ciências Rurais (CCR), Universidade Federal de Santa Maria (UFSM), 97105-900, Santa Maria, RS, Brasil. E-mail: cargnelutti@pq.cnpq.br. *Autor para correspondência.

"Programa de Pós-graduação em Agronomia, UFSM, Santa Maria, RS, Brasil.

${ }^{\text {IIIC }}$ Curso de Agronomia, UFSM, Santa Maria, RS, Brasil. 
confiança desejado na estimativa e inversamente proporcional ao erro de estimação permitido, fixado $a$ priori pelo pesquisador (FONSECA \& MARTINS, 1995; BARBETTAet al., 2004; BUSSAB \& MORETTIN, 2004; SPIEGEL et al., 2004). Em relação à estimativa da média de caracteres morfológicos e produtivos de crambe (Crambe abyssinica Hochst), não há um valor que possa ser definido como erro de estimação máximo tolerável. Porém, deve ser considerada a possibilidade de obter uma estimativa da média com a máxima confiabilidade possível.

Em um programa de melhoramento de plantas, o estudo das relações entre os caracteres é importante, a fim de identificar caracteres que possam ser utilizados na seleção indireta. No caso das relações existentes serem lineares, as análises de correlação, de trilha e de regressão linear multivariada possibilitam a obtenção de informações substanciais quanto à relação entre os caracteres.

$\mathrm{Na}$ cultura do crambe, planejamentos experimentais quanto ao tamanho e forma de parcela (MEIER \& LESSMAN, 1971) e relações entre caracteres (LESSMAN, 1975; MASTEBROEK etal., 1994) têm sido pesquisados. No entanto, informações sobre o tamanho de amostra e sobre relações entre caracteres morfológicos e produtivos de crambe ainda são escassos. Assim, os objetivos deste trabalho foram determinar o tamanho de amostra para a estimação da média de caracteres morfológicos e produtivos de crambe (Crambe abyssinica Hochst) e avaliar as relações entre esses caracteres.

\section{MATERIAL E MÉTODOS}

Foi realizado um experimento com a cultura de crambe (Crambe abyssinica Hochst) na Universidade Federal de Santa Maria, Santa Maria, Rio Grande do Sul (latitude $29^{\circ} 42^{\prime} \mathrm{S}$, longitude $53^{\circ} 49^{\prime} \mathrm{W}$ e $95 \mathrm{~m}$ de altitude), de julho a outubro de 2008. No mês de julho de 2008, foi efetuada a adubação de base na área experimental com $35 \mathrm{~kg} \mathrm{ha}^{-1}$ de N, $140 \mathrm{~kg} \mathrm{ha}^{-1} \mathrm{de}_{2} \mathrm{O}_{5} \mathrm{e}$ $140 \mathrm{~kg} \mathrm{ha}^{-1}$ de $\mathrm{K}_{2} \mathrm{O}$. Em seguida, foi feita a semeadura em 60 linhas de $20 \mathrm{~m}$ de comprimento e foi utilizado o espaçamento de $0,058 \mathrm{~m}$ entre plantas na linha e $0,17 \mathrm{~m}$ entre linhas, o que resulta em 100 plantas $\mathrm{m}^{-2}$.

$\mathrm{Na}$ área experimental, foram selecionadas, aleatoriamente, 75 plantas. Na colheita dessas plantas, foram mensurados os caracteres morfológicos: altura de planta em $\mathrm{cm}(\mathrm{AP})$, número de rácemos por planta
(NRP) e comprimento médio dos rácemos por planta em cm (CMRP). Também foram avaliados os caracteres produtivos: número de frutos por planta (NFP) e número de frutos por rácemo (NFR), obtidos dividindo-se NFP por NRP e produtividade de grãos, em gramas planta ${ }^{-1}$ (PROD). Em relação aos dados de AP, NRP, CMRP, NFP, NFR e PROD, foram calculadas medidas de tendência central, de variabilidade, de assimetria e curtose e verificada a normalidade, por meio do teste de Lilliefors (CAMPOS, 1983).

Para cada caráter, tomando-se por base as 75 plantas, foi calculado o tamanho de amostra ( $\eta$ ) para uma semiamplitude do intervalo de confiança (erro de estimação) igual a $1,2, \ldots$ e $40 \%$ da estimativa da média (m), com grau de confiança ( $1-\alpha$ ) de $95 \%$, por meio da

expressão $\left.\eta=\frac{\mathrm{t}_{\alpha / 2}^{2} \mathrm{~s}^{2}}{(\text { erro de estimação) }}\right)^{2}$ FONSECA\& MARTINS

1995; BARBETTAet al., 2004; BUSSAB \& MORETTIN, 2004; SPIEGEL et al., 2004), na qual t $t_{2}$ é o valor crítico da distribuição t de Student, cuja área à direita é igual a $\alpha / 2$, isto é, o valor de $t$, tal que $\mathrm{P}\left(\mathrm{t}>\mathrm{t}_{\alpha / 2}\right)=\alpha / 2$, com $(\mathrm{n}-1)$ graus de liberdade, $\operatorname{com} \alpha=5 \%$ de probabilidade de erro, e s $\mathrm{s}^{2}$ é a estimativa de variância.

Em seguida, fixando-se $\eta$ igual a 75 plantas, que foi o tamanho de amostra utilizado na amostragem, foi calculado o erro de estimação em percentagem da estimativa da média (m) para cada um dos caracteres,

por meio da expressão errode estimação $=100 \frac{\mathrm{t}_{\alpha / 2} \mathrm{~s}}{\sqrt{\eta} \mathrm{m}}, \mathrm{em}$ que s é a estimativa do desvio padrão.

A fim de visualizar as relações entre os caracteres AP, NRP, CMRP, NFP, NFR e PROD, foi feito o diagrama de dispersão. Após, foi calculada a matriz de coeficientes de correlação linear de Pearson (r) entre os caracteres e por meio do teste t de Student, a 5\% de probabilidade de erro, foi verificada a significância do r. A seguir, foi realizado o diagnóstico de multicolinearidade nessa matriz (CRUZ, 2006), conforme critério de MONTGOMERY \& PECK (1982). Depois disso, foi realizada a análise de trilha (path analysis) das variáveis explicativas (AP, NRP, CMRP e NFP) sobre a variável PROD e, em seguida, ajustado um modelo de regressão linear múltipla da variável dependente (PROD) em função das independentes (AP, NRP, CMRP e NFP). Os parâmetros do modelo foram estimados pelo método passo-a-passo (stepwise), com $5 \%$ de significância para entrada e saída de variáveis 
(CRUZ, 2006). O número de frutos por rácemo (NFR) foi excluído das análises de trilha e de regressão por causar multicolinearidade (MONTGOMERY \& PECK, 1982). As análises estatísticas foram realizadas com o auxílio do programa GENES (CRUZ, 2006) e do aplicativo Office Excel $\AA$.

\section{RESULTADOS E DISCUSSÃO}

De maneira geral, as medidas de tendência central, de variabilidade, de assimetria e curtose e o teste de Lilliefors, em relação aos caracteres morfológicos: altura de planta (AP), número de rácemos por planta (NRP) e comprimento médio dos rácemos por planta (CMRP), e aos produtivos: número de frutos por planta (NFP), número de frutos por rácemo (NFR) e produtividade de grãos (PROD), revelaram boa aderência dos dados, das 75 plantas de crambe (Crambe abyssinica Hochst), à distribuição normal (Tabela 1). Particularmente, os caracteres NFP e PROD apresentaram um leve afastamento da normalidade, caracterizado, principalmente, pela assimetria positiva da distribuição dos dados (Tabela 1 e Figura 1). No entanto, de acordo com o teorema limite central, mesmo que a população básica seja não normal, a distribuição da média amostral será aproximadamente normal para amostras superiores a 30 observações (FONSECA \& MARTINS, 1995; BUSSAB \& MORETTIN, 2004). Então, diante dessas considerações, em relação à normalidade, pode-se inferir que os dados desses caracteres oferecem credibilidade ao estudo do dimensionamento de amostra.

A magnitude do coeficiente de variação (CV) oscilou entre $19,54 \%$ para a altura de planta e $62,29 \%$ para o número de frutos por planta. De maneira geral, $o$ $\mathrm{CV}$ dos caracteres produtivos (média dos três caracteres $=56,77 \%$ ), foi, aproximadamente, 2,4 vezes maior que o $\mathrm{CV}$ dos caracteres morfológicos (média dos três caracteres $=23,78 \%$ ). Isso sugere que para a obtenção da estimativa da média, com uma determinada precisão, o tamanho de amostra de caracteres produtivos é maior em relação aos morfológicos. Ainda, a variabilidade existente é importante e confere adequabilidade ao estudo das relações entre os caracteres, por meio das análises de correlação, de trilha e de regressão.

O tamanho de amostra (número de plantas) para a estimação da média de cada caráter, com semiamplitude do intervalo de confiança igual a $1 \%$ da estimativa da média (m) e grau de confiança de $95 \%$, oscilou entre 1.516 plantas, para o caráter altura de planta; a 15.405 plantas para o caráter número de frutos por planta (Tabela 2). Portanto, em relação altura de planta, pode-se inferir, com $95 \%$ de confiança, que o intervalo de confiança da média $(\mu)$, obtido com 1.516 plantas, é de $\mu \pm 0,01 \mathrm{~m}$. Em outro extremo, a precisão de $\mu \pm 0,01 \mathrm{~m}$ é obtida com 15.405 plantas, para o número de frutos por planta. Esses resultados confirmam a variabilidade existente entre os caracteres. A

Tabela 1 - Mínimo, máximo, média, mediana, desvio-padrão, erro-padrão da média, coeficiente de variação (CV), assimetria, curtose e teste de Lilliefors da altura de planta (AP), em cm; do número de rácemos por planta (NRP), do comprimento médio dos rácemos por planta (CMRP), em cm; do número de frutos por planta (NFP), do número de frutos por rácemo (NFR) e da produtividade de grãos (PROD), em gramas planta ${ }^{-1}$ de 75 plantas de crambe (Crambe abyssinica Hochst).

\begin{tabular}{|c|c|c|c|c|c|c|}
\hline Estatística & AP & NRP & CMRP & NFP & NFR & PROD \\
\hline Mínimo & 31,00000 & 4,00000 & 7,90000 & 26,00000 & 3,25000 & 0,13670 \\
\hline Máximo & 92,30000 & 19,00000 & 30,48571 & 535,00000 & 29,87500 & 3,41640 \\
\hline Média & 65,69467 & 11,17333 & 19,96338 & 186,32000 & 15,85630 & 1,18392 \\
\hline Mediana & 65,50000 & 11,00000 & 19,36923 & 173,00000 & 15,90909 & 1,08870 \\
\hline Desvio-padrão & 12,83450 & 2,79175 & 5,35658 & 116,05825 & 7,30529 & 0,73352 \\
\hline Erro-padrão & 1,48200 & 0,32236 & 0,61852 & 13,40125 & 0,84354 & 0,08470 \\
\hline $\mathrm{CV}(\%)$ & 19,53659 & 24,98584 & 26,83203 & 62,28974 & 46,07185 & 61,95716 \\
\hline Assimetria $^{(1)}$ & $-0,16476 \mathrm{~ns}$ & $0,18039 \mathrm{~ns}$ & $-0,01002 \mathrm{~ns}$ & $0,98010 *$ & $0,25064 \mathrm{~ns}$ & $0,92429 *$ \\
\hline Curtose $+3^{(2)}$ & $2,71832 \mathrm{~ns}$ & $3,25082 \mathrm{~ns}$ & $2,09478 \mathrm{~ns}$ & $3,64077 \mathrm{~ns}$ & $2,11606 \mathrm{~ns}$ & $3,48343 \mathrm{~ns}$ \\
\hline Lilliefors ${ }^{(3)}$ & $0,06456 \mathrm{~ns}$ & $0,10357 *$ & $0,07374 \mathrm{~ns}$ & $0,09928 \mathrm{~ns}$ & $0,09527 \mathrm{~ns}$ & $0,11887 *$ \\
\hline
\end{tabular}

(1) * Assimetria difere de zero pelo teste $\mathrm{t}$, em nível de $5 \%$ de probabilidade. $\mathrm{ns}=$ não-significativo.

(2) * Curtose difere de três pelo teste $\mathrm{t}$, em nível de $5 \%$ de probabilidade. $\mathrm{ns}=$ não-significativo.

(3) * Significativo a $5 \%$ de probabilidade. ns = não-significativo. 


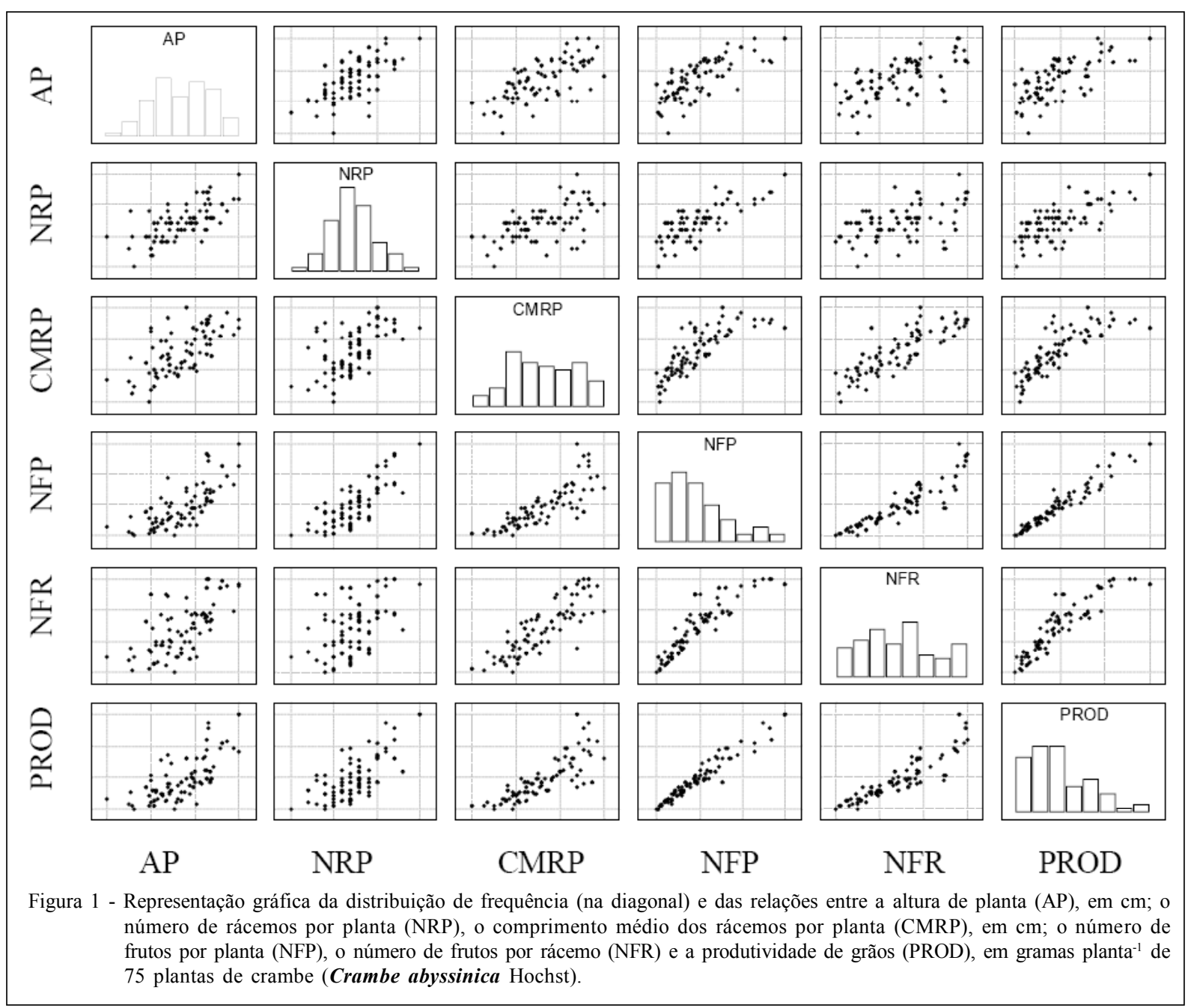

variabilidade de tamanho de amostra entre genótipos, caracteres e experimentos foi constatada em milho (STORCK et al., 2007), em feijão (CARGNELUTTI FILHO et al., 2008), em soja (CARGNELUTTI FILHO et al., 2009) e em mamona (CARGNELUTTI FILHO et al., 2010).

Do ponto de vista prático, pode-se inferir que é difícil obter estimativas da média de caracteres morfológicos e produtivos de crambe, com erro de estimação de $1 \%$, em função do elevado número de plantas a serem mensuradas. Por exemplo, para o caráter altura de planta, que apresenta menor variabilidade, seriam necessárias 1.516 plantas. Assim, menores tamanhos de amostra foram determinados com base em semiamplitudes do intervalo de confiança iguais a $2,3, \ldots$ e $40 \%$ da média (m) (Tabela 2 ). Não cabe aqui o julgamento do erro de estimação máximo aceitável, ficando isso a critério do pesquisador que usufruir dessas informações para o seu planejamento experimental. Caso a opção seja amostrar 75 plantas, $o$ erro de estimação seria de $\pm 4,49, \pm 5,75, \pm 6,17, \pm 14,33$, $\pm 10,60$ e $\pm 14,26 \%$ da estimativa da média (m), respectivamente, para os caracteres AP, NRP, CMRP, NFP, NFR e PROD. Portanto, os resultados evidenciam que o tamanho de amostra de caracteres produtivos é maior que o de morfológicos. Resultados semelhantes foram obtidos na cultura da mamona, por CARGNELUTTI FILHO et al. (2010), que concluíram que há aumento do tamanho de amostra (número de plantas) para estimar caracteres de semente, de plântula, de planta adulta e de produção, nessa ordem.

$\mathrm{O}$ diagnóstico de multicolinearidade na matriz de coeficientes de correlação linear de Pearson, entre as variáveis explicativas altura de planta (AP), número de rácemos por planta (NRP), comprimento médio dos rácemos por planta (CMRP), número de frutos por planta (NFP) e número de frutos por rácemo (NFR), revelou número de condição (NC) igual a 228, o 
Tabela 2 - Tamanho de amostra (número de plantas) para a estimação da média dos caracteres altura de planta (AP), número de rácemos por planta (NRP), comprimento médio dos rácemos por planta (CMRP), número de frutos por planta (NFP), número de frutos por rácemo (NFR) e produtividade de grãos (PROD) de crambe (Crambe abyssinica Hochst), para uma semiamplitude do intervalo com $95 \%$ de confiança e erros iguais a $1,2, \ldots, 40 \%$ da média.

\begin{tabular}{|c|c|c|c|c|c|c|}
\hline Erro (\%) & $\mathrm{AP}$ & NRP & CMRP & NFP & NFR & PROD \\
\hline 1 & 1516 & 2479 & 2859 & 15405 & 8428 & 15241 \\
\hline 2 & 379 & 620 & 715 & 3852 & 2107 & 3811 \\
\hline 3 & 169 & 276 & 318 & 1712 & 937 & 1694 \\
\hline 4 & 95 & 155 & 179 & 963 & 527 & 953 \\
\hline 5 & 61 & 100 & 115 & 617 & 338 & 610 \\
\hline 6 & 43 & 69 & 80 & 428 & 235 & 424 \\
\hline 7 & 31 & 51 & 59 & 315 & 172 & 312 \\
\hline 8 & 24 & 39 & 45 & 241 & 132 & 239 \\
\hline 9 & 19 & 31 & 36 & 191 & 105 & 189 \\
\hline 10 & 16 & 25 & 29 & 155 & 85 & 153 \\
\hline 11 & 13 & 21 & 24 & 128 & 70 & 126 \\
\hline 12 & 11 & 18 & 20 & 107 & 59 & 106 \\
\hline 13 & 9 & 15 & 17 & 92 & 50 & 91 \\
\hline 14 & 8 & 13 & 15 & 79 & 43 & 78 \\
\hline 15 & 7 & 12 & 13 & 69 & 38 & 68 \\
\hline 16 & 6 & 10 & 12 & 61 & 33 & 60 \\
\hline 17 & 6 & 9 & 10 & 54 & 30 & 53 \\
\hline 18 & 5 & 8 & 9 & 48 & 27 & 48 \\
\hline 19 & 5 & 7 & 8 & 43 & 24 & 43 \\
\hline 20 & 4 & 7 & 8 & 39 & 22 & 39 \\
\hline 21 & 4 & 6 & 7 & 35 & 20 & 35 \\
\hline 22 & 4 & 6 & 6 & 32 & 18 & 32 \\
\hline 23 & 3 & 5 & 6 & 30 & 16 & 29 \\
\hline 24 & 3 & 5 & 5 & 27 & 15 & 27 \\
\hline 25 & 3 & 4 & 5 & 25 & 14 & 25 \\
\hline 26 & 3 & 4 & 5 & 23 & 13 & 23 \\
\hline 27 & 3 & 4 & 4 & 22 & 12 & 21 \\
\hline 28 & 2 & 4 & 4 & 20 & 11 & 20 \\
\hline 29 & 2 & 3 & 4 & 19 & 11 & 19 \\
\hline 30 & 2 & 3 & 4 & 18 & 10 & 17 \\
\hline 31 & 2 & 3 & 3 & 17 & 9 & 16 \\
\hline 32 & 2 & 3 & 3 & 16 & 9 & 15 \\
\hline 33 & 2 & 3 & 3 & 15 & 8 & 14 \\
\hline 34 & 2 & 3 & 3 & 14 & 8 & 14 \\
\hline 35 & 2 & 3 & 3 & 13 & 7 & 13 \\
\hline 36 & 2 & 2 & 3 & 12 & 7 & 12 \\
\hline 37 & 2 & 2 & 3 & 12 & 7 & 12 \\
\hline 38 & 2 & 2 & 2 & 11 & 6 & 11 \\
\hline 39 & 1 & 2 & 2 & 11 & 6 & 11 \\
\hline 40 & 1 & 2 & 2 & 10 & 6 & 10 \\
\hline
\end{tabular}

que classifica a matriz como de colinearidade moderada a forte, conforme critério de MONTGOMERY \& PECK (1982). Com a retirada do NFR, o NC reduziu para 24 e, assim, as análises de trilha e de regressão linear múltipla da produtividade de grãos (PROD) em função das variáveis explicativas (AP, NRP, CMRP e NFP) podem ser realizadas adequadamente (CRUZ, 2006) (Tabela 3).

A produtividade de grãos (PROD) apresentou correlação linear positiva $(\mathrm{r}=0,96523, \mathrm{P} \leq 0,05)$ e efeito direto $(0,97729)$ com mesmo sinal e de magnitude semelhante com o número de frutos por planta (NFP), explicando a verdadeira associação existente. Assim, pode-se inferir que as plantas com maior número de frutos são aquelas com maior produtividade de grãos. Em menor magnitude, a PROD apresentou associação linear com a AP $(r=0,69596$, $\mathrm{P} \leq 0,05)$, o NRP $(\mathrm{r}=0,69368, \mathrm{P} \leq 0,05)$ e o $\mathrm{CMRP}(\mathrm{r}=0,81797$, $\mathrm{P} \leq 0,05)$. No entanto, os efeitos diretos da AP $(-0,00787)$, do NRP $(-0,08449)$ e do CMRP $(0,07073)$ sobre a PROD foram desprezíveis e, portanto, a associação existente é explicada pelos elevados efeitos indiretos via NFP $(\mathrm{AP}=0,71348 ; \mathrm{NRP}=0,74314$ e $\mathrm{CMRP}=0,80049)$ (Tabela $3)$. Esses resultados sugerem que a seleção indireta para a PROD deve ser realizada com base no NFP.

A estimativa da equação de regressão linear múltipla, obtida por meio do método stepwise, da PROD foi função somente do NFP (PROD=0,047262+0,006101 NFP) e apresentou coeficiente de determinação $\left(\mathrm{R}^{2}\right)$ de 0,9317. A não inclusão das variáveis explicativas AP, NRP e CMRP, nesse modelo, é explicada pelos efeitos diretos desprezíveis e elevados efeitos indiretos via NFP sobre a PROD (Tabela 3). Então, diante desses resultados, confirma-se a relação linear positiva existente entre o NFP e a PROD e, portanto, o NFP é um caráter adequado para a seleção indireta quanto a PROD. Do ponto de vista prático, é possível selecionar as plantas diretamente na área experimental, por meio da contagem do número de frutos, sem a necessidade de destruir as plantas para a colheita.

\section{CONCLUSÃO}

Na cultura de crambe (Crambe abyssinica Hochst), para estimar a média com uma mesma precisão, o tamanho de amostra (número de plantas) dos caracteres produtivos deve ser maior do que o tamanho dos morfológicos. O número de frutos por planta tem relação linear positiva com a produtividade de grãos e pode ser utilizado para seleção indireta.

\section{AGRADECIMENTOS}

Ao Conselho Nacional de Desenvolvimento Científico e Tecnológico (CNPq) e à Coordenação de Aperfeiçoamento de Pessoal de Nível Superior (CAPES), pelas bolsas concedidas.

Ciência Rural, v.40, n.11, nov, 2010. 
Tabela 3 - Estimativas dos coeficientes de correlação linear de Pearson e dos efeitos diretos e indiretos dos caracteres altura de planta (AP), número de rácemos por planta (NRP), comprimento médio dos rácemos por planta (CMRP) e número de frutos por planta (NFP) sobre a produtividade de grãos (PROD) de 75 plantas de crambe (Crambe abyssinica Hochst).

\begin{tabular}{llcc}
\hline Efeito & AP & NRP & CMRP \\
\hline Direto sobre PROD & $-0,00787$ & $-0,08449$ & 0,07073 \\
Indireto via AP & & $-0,00522$ & $-0,00516$ \\
Indireto via NRP & $-0,05606$ & $-0,04809$ \\
Indireto via CMRP & 0,04639 & 0,04025 & 0,00049 \\
Indireto via NFP & 0,71348 & 0,74314 & 0,05793 \\
Total (Correlação de Pearson) & $0,69596^{*}$ & $0,69368^{*}$ & $0,81797^{*}$ \\
Coeficiente de determinação & 0,93708 & & $0,96523^{*}$ \\
Efeito da variável residual & 0,25083 & & \\
\hline
\end{tabular}

* Significativo a $5 \%$ de probabilidade de erro pelo teste t, com 73 graus de liberdade.

\section{REFERÊNCIAS}

BARBETTA, P.A. et al. Estatística para cursos de engenharia e informática. São Paulo: Atlas, 2004. 410p.

BUSSAB, W.O.; MORETTIN, P.A. Estatística básica. 5.ed. São Paulo: Saraiva, 2004. 526p.

CAMPOS, H. De. Estatística experimental nãoparamétrica. 4.ed. Piracicaba: Departamento de Matemática e Estatística - ESALQ, 1983. 349p.

CARGNELUTTI FILHO, A. et al. Tamanho de amostra de caracteres de genótipos de soja. Ciência Rural, v.39, p.983991, 2009. Disponível em: <http://www.scielo.br/ scielo.php?script=sci_arttext\&pid=S 0103 $84782009000400005 \& \operatorname{lng}=\mathrm{pt} \& \mathrm{nrm}=\mathrm{iso}>$. Acesso em: $18 \mathrm{mar}$. 2010. doi: $10.1590 / \mathrm{S} 0103-84782009005000016$.

CARGNELUTTI FILHO, A. et al. Tamanho de amostra de caracteres de cultivares de feijão. Ciência Rural, v.38, p.635-642, 2008. Disponível em: $<$ http://www.scielo.br/scielo.php?script=sci_arttext\&pid=S0103$84782008000300007 \& \operatorname{lng}=$ pt\&nrm=iso>. Acesso em: $18 \mathrm{mar}$. 2010. doi: $10.1590 / \mathrm{S} 0103-84782008000300007$.

CARGNELUTTI FILHO, A. et al. Tamanho de amostra de caracteres em híbridos de mamoneira. Ciência Rural, v.40, p.250-257, 2010. Disponível em: <http://www.scielo.br/ scielo.php?script=sci_arttext\&pid=S 0103 $84782010000200005 \& \operatorname{lng}=\mathrm{pt \& nrm}=\mathrm{iso}>$. Acesso em: $18 \mathrm{mar}$. 2010 .
CRUZ, C.D. Programa genes: estatística experimental e matrizes. Viçosa: UFV, 2006. 285p.

FONSECA, J.S.; MARTINS, G.A. Curso de estatística. 5.ed. São Paulo: Atlas, 1995. 317p.

LESSMAN, K.J. Variation in crambe, Crambe abyssinica Hochst. Journal of the American Oil Chemists' Society, v.52, p.386-389, 1975.

MASTEBROEK, H.D. et al. Variation for agronomic characteristics in crambe (Crambe abyssinica Hochst. ex Fries). Industrial Crops and Products, v.2, p.129-136, 1994.

MEIER, V.D.; LESSMAN, K.J. Estimation of optimum field plot shape and size for testing yield in Crambe abyssinica Hochst. Crop Science, v.11, p.648-650, 1971.

MONTGOMERY, D.C.; PECK, E.A. Introduction to linear regression analysis. New York: John Wiley \& Sons, 1982. $504 \mathrm{p}$.

SPIEGEL, R.A. et al. Probabilidade e estatística. 2.ed. Porto Alegre: Bookman, 2004. 398p.

STORCK, L. et al. Sample size for single, double and triple hybrid corn ear traits. Scientia Agrícola, v.64, p. 30-35, 2007. Disponível $\mathrm{em}:<\mathrm{http}: / /$ www.scielo.br/scielo.php?script=sci_arttext\&pid=S010390162007000100005\&lng=pt\&nrm=iso>. Acesso em: 18 mar. 2010. doi: $10.1590 / \mathrm{S} 0103-90162007000100005$. 